DOE-ER-40757-093

UTEXAS-HEP-97-2

MSUHEP-70205

\title{
Photon-neutrino interactions
}

\author{
Duane A. Dicus \\ Center for Particle Physics and Department of Physics \\ University of Texas, Austin, Texas 78712 \\ Wayne W. Repko \\ Department of Physics and Astronomy \\ Michigan State University, East Lansing, Michigan 48824
}

(July 16, 2018)

\begin{abstract}
The cross sections for the processes $\gamma \nu \rightarrow \gamma \gamma \nu, \gamma \gamma \rightarrow \gamma \nu \bar{\nu}$ and $\nu \bar{\nu} \rightarrow \gamma \gamma \gamma$ are calculated with the aid of an effective Lagrangian derived from the Standard model. These cross sections are shown to be much larger than the elastic cross section $\sigma(\gamma \nu \rightarrow \gamma \nu)$ for photon energies $\omega>1 \mathrm{keV}$. Possible astrophysical implications are discussed.
\end{abstract}

13.10.+q, 14.60.Gh,14.80.Am, 95.30.Cq

\section{ELASTIC PROCESSES}

Photon-neutrino scattering is of potential interest in astrophysical processes. The various $\gamma \nu$ reaction cross sections, which are expected to be small due to the weak interaction, are further suppressed because of the vector-axial vector nature of the weak coupling [1] 3]. For the channel $\nu \bar{\nu} \rightarrow \gamma \gamma$, Yang's theorem [4] implies a vanishing decay amplitude to order $G_{F}$ when the neutrinos are massless. This means that the amplitude in any channel contains additional factors of $\omega / m_{W}$, where $\omega$ is the photon energy and $m_{W}$ is the $W$ mass. If the neutrinos have a mass $m_{\nu}$, the amplitude is reduced by factors of $m_{\nu} / m_{W}$ [5].

In the case of massless neutrinos, the amplitude for $\gamma \nu \rightarrow \gamma \nu$ in the Standard model is $[6]$, to leading order in $m_{W}^{-2}$,

$$
\mathcal{M}_{\lambda \lambda^{\prime}}=\frac{1}{2 \pi} \frac{g^{2} \alpha}{m_{W}^{4}}\left[1+\frac{4}{3} \ln \left(\frac{m_{W}^{2}}{m_{e}^{2}}\right)\right] \cos (\theta / 2)\left(s^{2}\left(1+\lambda \lambda^{\prime}\right)+\frac{s t}{2}(1+\lambda)\left(1+\lambda^{\prime}\right)\right),
$$

where $\lambda$ and $\lambda^{\prime}$ are the photon helicities, $s=4 \omega^{2}, t=-2 \omega^{2}(1-\cos \theta)$ and $\theta$ is the scattering angle. Notice that there are no inverse powers of $m_{e}$ in this expression and hence the scale is set by $m_{W}$. As a consequence, all $2 \rightarrow 2$ $\gamma \nu$ channels can be calculated using the effective Lagrangian

$$
\mathcal{L}_{\mathrm{eff}}=\frac{1}{4 \pi} \frac{g^{2} \alpha}{m_{W}^{4}}\left[1+\frac{4}{3} \ln \left(\frac{m_{W}^{2}}{m_{e}^{2}}\right)\right]\left[\bar{\psi} \gamma_{\nu}\left(1+\gamma_{5}\right)\left(\partial_{\mu} \psi\right)-\left(\partial_{\mu} \bar{\psi}\right) \gamma_{\nu}\left(1+\gamma_{5}\right) \psi\right] F_{\mu \lambda} F_{\nu \lambda}
$$

where $F_{\mu \nu}$ is the photon field tensor and $\psi$ is the neutrino field. The resulting cross section for elastic scattering is

$$
\sigma(\gamma \nu \rightarrow \gamma \nu)=\frac{3}{4} \frac{G_{F}^{2} \alpha^{2}}{\pi^{3}}\left[1+\frac{4}{3} \ln \left(\frac{m_{W}^{2}}{m_{e}^{2}}\right)\right]^{2}\left(\frac{\omega}{m_{W}}\right)^{4} \omega^{2} .
$$

Despite the enhancement of the $\ln ^{2}\left(m_{W}^{2} / m_{e}^{2}\right)$ factor, this cross section is exceedingly small and likely to be of little practical importance in astrophysics.

\section{INELASTIC PROCESSES}

The source of the large suppression in the $\gamma \nu$ elastic amplitude is the Yang theorem prohibition of a two photon coupling to a $J=1$ state. There is no similar restriction on the coupling of three photons. This suggests an examination of the inelastic process $\gamma \nu \rightarrow \gamma \gamma \nu$ to determine if the scale of the loop integrals resulting from the $W$ 
and $Z$ exchange diagrams represented by Fig. 1 is set by the electron mass rather than $m_{W}$. This turns out to be the case, and one can obtain an effective Lagrangian of the form

$$
\mathcal{L}_{\mathrm{eff}}=4 \frac{G_{F} a}{\sqrt{2}} \frac{\alpha^{3 / 2}}{\sqrt{4 \pi}} \frac{1}{m_{e}^{4}}\left[\frac{5}{180}\left(N_{\mu \nu} F_{\mu \nu}\right)\left(F_{\lambda \rho} F_{\lambda \rho}\right)-\frac{14}{180} N_{\mu \nu} F_{\nu \lambda} F_{\lambda \rho} F_{\rho \mu}\right],
$$

where $N_{\mu \nu}$ is

$$
N_{\mu \nu}=\partial_{\mu}\left(\bar{\psi} \gamma_{\nu}\left(1+\gamma_{5}\right) \psi\right)-\partial_{\nu}\left(\bar{\psi} \gamma_{\mu}\left(1+\gamma_{5}\right) \psi\right)
$$

and $a=1-\frac{1}{2}\left(1-4 \sin ^{2} \theta_{W}\right)$. The numerical factors 5/180 and -14/180 are familiar from the Euler-Heisenberg $[7]$ expansion of the photon-photon scattering amplitude. This occurs because after Fierz rearranging the $W$ contribution and replacing the gauge boson propagators by their masses, the amplitude in any channel for either $W$ or $Z$ exchange reduces to a four photon amplitude with one photon polarization vector replaced by the neutrino current. The $Z$ contribution, given by the second factor in $a$, is numerically small $\approx .04$. Using Eq. (A), the double differential cross section $d^{2} \sigma / d \omega_{1} d \omega_{2}$ for the energy distribution of the final photons in the neutrino-photon center of mass is

$$
\begin{aligned}
\frac{d^{2} \sigma}{d \omega_{1} d \omega_{2}}= & \frac{G_{F}^{2} a^{2} \alpha^{3}}{\pi^{4} m_{e}^{8}} \frac{\omega^{3}}{30,375}\left[-\frac{1}{2}\left(3614 \omega_{1}^{2} \omega_{2}^{2}\left(\omega_{1}+\omega_{2}\right)+2085 \omega_{1} \omega_{2}\left(\omega_{1}^{3}+\omega_{2}^{3}\right)\right.\right. \\
& \left.+695\left(\omega_{1}^{5}+\omega_{2}^{5}\right)\right)+\omega\left(5372 \omega_{1} \omega_{2}\left(\omega_{1}^{2}+\omega_{2}^{2}\right)+8103 \omega_{1}^{2} \omega_{2}^{2}+2085\left(\omega_{1}^{4}+\omega_{2}^{4}\right)\right) \\
& -\omega^{2}\left(15,552 \omega_{1} \omega_{2}\left(\omega_{1}+\omega_{2}\right)+6067\left(\omega_{1}^{3}+\omega_{2}^{3}\right)\right)+\omega^{3}\left(21,750 \omega_{1} \omega_{2}\right. \\
& \left.\left.+11,063\left(\omega_{1}^{2}+\omega_{2}^{2}\right)\right)-\frac{21,055}{2} \omega^{4}\left(\omega_{1}+\omega_{2}\right)+3794 \omega^{5}\right] .
\end{aligned}
$$

The cross section for $\gamma \nu \rightarrow \gamma \gamma \nu$ can be obtained by integrating Eq. (6) over the region $\left(\omega-\omega_{1}\right) \leq \omega_{2} \leq \omega, 0 \leq \omega_{1} \leq \omega$ and is

$$
\sigma(\gamma \nu \rightarrow \gamma \gamma \nu)=\frac{262}{127,575} \frac{G_{F}^{2} a^{2} \alpha^{3}}{\pi^{4}}\left(\frac{\omega}{m_{e}}\right)^{8} \omega^{2}
$$

Further details of the scattering process are presented in Fig. 2. By retaining the polarization vector of one of the final state photons, it is possible to obtain the cross section for this photon to be produced with either positive or negative helicity. This is illustrated in the left panel of Fig. 2 by the dashed and dot-dashed lines. The solid line in this panel is the angular distribution for unpolarized scattering and $\theta$ is the angle between the outgoing photon and the incident photon. The difference between the positive and negative helicity cross sections, which are fifth order polynomials in $\cos \theta$, results in the polarization $P(\theta)$ illustrated in the right panel of Fig. 2 . The existence of a net circular polarization is possible because the weak interaction violates parity.

It is also of interest to examine the annihilation channel $\gamma \gamma \rightarrow \gamma \nu \bar{\nu}$, which provides an energy loss mechanism for stellar processes [8 10]. In this case, the double differential cross section $d^{2} \sigma / \sin \theta d \theta d \omega^{\prime}$ is

$$
\frac{d^{2} \sigma}{\sin \theta d \theta d \omega^{\prime}}=\frac{G_{F}^{2} a^{2} \alpha^{3}}{\pi^{4} m_{e}^{8}} \frac{\omega^{3} \omega^{\prime 3}\left(\omega-\omega^{\prime}\right)}{48,600}\left[2224 \omega^{2}-\omega\left(592 \omega+520 \omega^{\prime}\right) \sin ^{2} \theta+139 \omega^{\prime 2} \sin ^{4} \theta\right]
$$

where $\theta$ is the scattering angle of the final photon in the center of mass, $\omega^{\prime}$ is its energy and $\omega$ is the initial photon energy. When integrated, Eq. (8) gives the total cross section

$$
\sigma(\gamma \gamma \rightarrow \gamma \nu \bar{\nu})=\frac{2,144}{637,875} \frac{G_{F}^{2} a^{2} \alpha^{3}}{\pi^{4}}\left(\frac{\omega}{m_{e}}\right)^{8} \omega^{2}
$$

Unlike $\gamma \nu \rightarrow \gamma \gamma \nu$, the final photon in the annihilation channel does not acquire any circular polarization.

The neutrino annihilation channel $\nu \bar{\nu} \rightarrow \gamma \gamma \gamma$ is potentially important in supernova calculations. Here, the energy distribution of two of the final photons in the neutrino center of mass is

$$
\begin{aligned}
\frac{d^{2} \sigma}{d \omega_{1} d \omega_{2}}= & \frac{G_{F}^{2} a^{2} \alpha^{3}}{\pi^{4} m_{e}^{8}} \frac{8 \omega^{4}}{18,225}\left[139\left(\left(\omega_{1}^{4}+\omega_{2}^{4}\right)+3 \omega_{1}^{2} \omega_{2}^{2}+2 \omega_{1} \omega_{2}\left(\omega_{1}^{2}+\omega_{2}^{2}\right)\right)\right. \\
& -4 \omega\left(139\left(\omega_{1}^{3}+\omega_{2}^{3}\right)+315 \omega_{1} \omega_{2}\left(\omega_{1}+\omega_{2}\right)\right)+2 \omega^{2}\left(491\left(\omega_{1}^{2}+\omega_{2}^{2}\right)+917 \omega_{1} \omega_{2}\right) \\
& \left.-852 \omega^{3}\left(\omega_{1}+\omega_{2}\right)+287 \omega^{4}\right],
\end{aligned}
$$


which may be integrated to give

$$
\sigma(\nu \bar{\nu} \rightarrow \gamma \gamma \gamma)=\frac{136}{91,125} \frac{G_{F}^{2} a^{2} \alpha^{3}}{\pi^{4}}\left(\frac{\omega}{m_{e}}\right)^{8} \omega^{2}
$$

\section{DISCUSSION AND CONCLUSIONS}

A direct comparison of the elastic and inelastic cross sections, Eqs. (3), (7), (9) and (11), is given in Table 1. The $\omega^{10}$ behavior of the inelastic cross sections versus the $\omega^{6}$ behavior of the elastic cross section is evident as is the 14 orders of magnitude difference at $\omega=2 m_{e}$. Certainly, the effective Lagrangian Eq. (4) provides an adequate description of the inelastic processes for $\omega<m_{e}$. The application of Eq. (8) to stellar energy loss is therefore completely justified. At some point beyond $\omega=m_{e}$, the cross section ceases to grow as the tenth power, begins a transition to a 'soft' behavior and eventually decreases. The precise range of applicability of the power law is somewhat subjective in the sense that numerical factors resulting from the loop integrals which define $\mathcal{L}_{\text {eff }}$ are often included in the definition of the scale factor. Including the factor of 180 appearing in Eq. (田), the effective scale is $\sim 4 m_{e}$.

As a rough indication of the importance of the $\gamma \nu \rightarrow \gamma \gamma \nu$ process in cosmology, consider the mean number of collisions in an expansion time $t$. Assuming that there is an effective scale greater than $m_{e}$, Eq. (7) can be written

$$
\sigma(\gamma \nu \rightarrow \gamma \gamma \nu)=2.0 \times 10^{-53} T_{10}^{10} \mathrm{~cm}^{2}
$$

where $T_{10}$ is the photon energy in units of $10^{10} \mathrm{~K}$ and $\omega \sim T$. The mean number of collisions in this time [11], $\sigma(\gamma \nu \rightarrow \gamma \gamma \nu) n_{\nu} c t$, where $n_{\nu}$ denotes the neutrino density, is

$$
\sigma(\gamma \nu \rightarrow \gamma \gamma \nu) n_{\nu} c t=1.92 \times 10^{-11} T_{10}^{11}
$$

For this to be of order $1, T_{10} \sim 9.4$ or $T \sim 15 m_{e}$. Strictly speaking, this result is beyond the scale $\sim 4 m_{e}$, and it is probably necessary to treat the transition region more carefully in order to determine the decoupling temperature. If this temperature were shown to be low enough, the process $\gamma \nu \rightarrow \gamma \gamma \nu$ might be of some importance in cosmological considerations. There is the possibility, albeit remote, that the detection of circular polarization, which only occurs in parity violating processes, could provide evidence for relic neutrino interactions.

\section{ACKNOWLEDGMENTS}

We would like to thank V. Teplitz, M. Einhorn and V. Zelevinsky for helpful conversations. We are indebted to A. V. Kuznetsov and N. V. Mikheev for drawing our attention to Ref. [9] and informing us of their work. This research was supported in part by the U.S. Department of Energy under Contract No. DE-FG013-93ER40757 and in part by the National Science Foundation under Grant No. PHY-93-07980.

[1] M. J. Levine, Nuovo Cimento 48A, 67 (1967).

[2] V. K. Cung and M. Yoshimura, Nuovo Cimento 29A, 557 (1975).

[3] J. Liu, Phys. Rev. D 44, 2879 (1991).

[4] C. N. Yang, Phys. Rev. 77, 242 (1950); L. D. Landau, Sov. Phys. Doklady 60, 207 (1948).

[5] M. Gell-Mann, Phys. Rev. Lett. 6, 70 (1961).

[6] D. A. Dicus and W. W. Repko, Phys. Rev. D 48, 5106 (1993).

[7] H. Euler, Ann. Phys. 26, 398 (1936); W. Heisenberg and H. Euler, Zeit. Phys. 98, 714 (1936).

[8] H.-Y. Chiu and P. Morrison, Phys. Rev. Lett. 5, 573 (1960).

[9] N. Van Hieu and E. P. Shabalin, Sov. Phys. JETP 17, 681 (1963). Our result, Eq. (8), for the double differential cross section differs from the one given in this paper.

[10] A. V. Kuznetsov and N. V. Mikheev (unpublished).

[11] P. J. E. Peebles, Principles of Physical Cosmology, Princeton University Press, Princeton, New Jersey (1993). 
TABLE I. $\sigma(\omega)$ in $\mathrm{cm}^{2}$ for $\omega$ in $\mathrm{keV}$

\begin{tabular}{|c|r|r|r|r|}
\hline \hline \hline & $\sigma(\gamma \nu \rightarrow \gamma \gamma \nu)$ & $\sigma(\gamma \gamma \rightarrow \gamma \nu \bar{\nu})$ & $\sigma(\nu \bar{\nu} \rightarrow \gamma \gamma \gamma)$ & \\
\hline 1 & $8.66 \times 10^{-83}$ & $1.42 \times 10^{-82}$ & $6.29 \times 10^{-83}$ & \\
100 & $8.66 \times 10^{-63}$ & $1.42 \times 10^{-62}$ & $6.29 \times 10^{-63}$ & $2.05 \times 10^{-84}$ \\
511 & $1.05 \times 10^{-55}$ & $1.72 \times 10^{-55}$ & $7.63 \times 10^{-56}$ & $2.05 \times 10^{-72}$ \\
1022 & $1.08 \times 10^{-52}$ & $1.77 \times 10^{-52}$ & $7.85 \times 10^{-53}$ & $3.65 \times 10^{-68}$ \\
\hline \hline
\end{tabular}

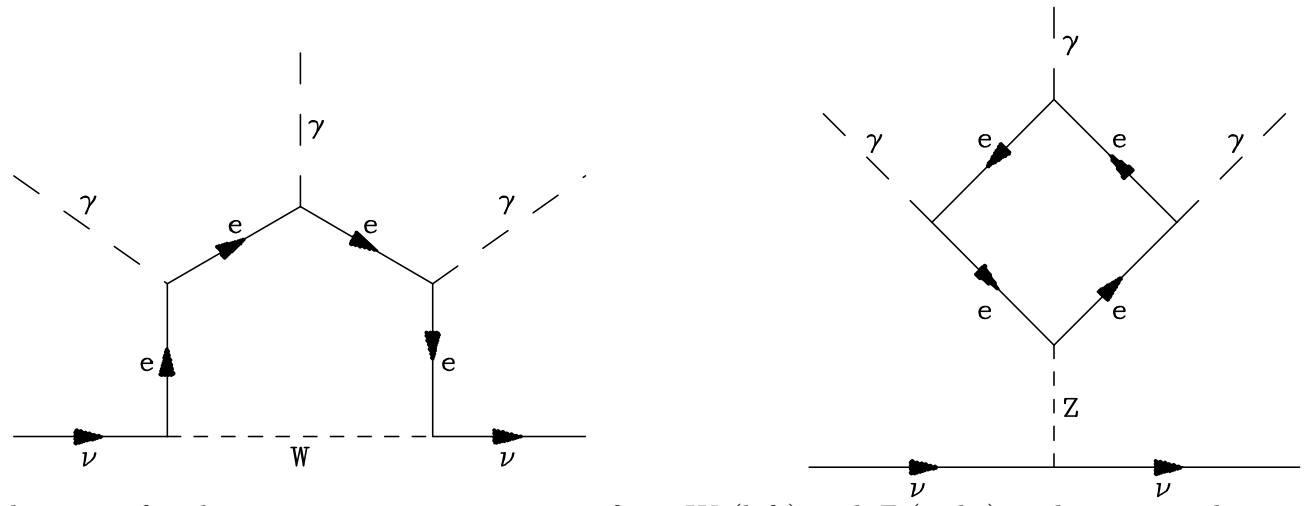

FIG. 1. Typical diagrams for the process $\gamma \nu \rightarrow \gamma \gamma \nu$ arising from $W$ (left) and $Z$ (right) exchange are shown. The complete set is obtained by permuting the photons.
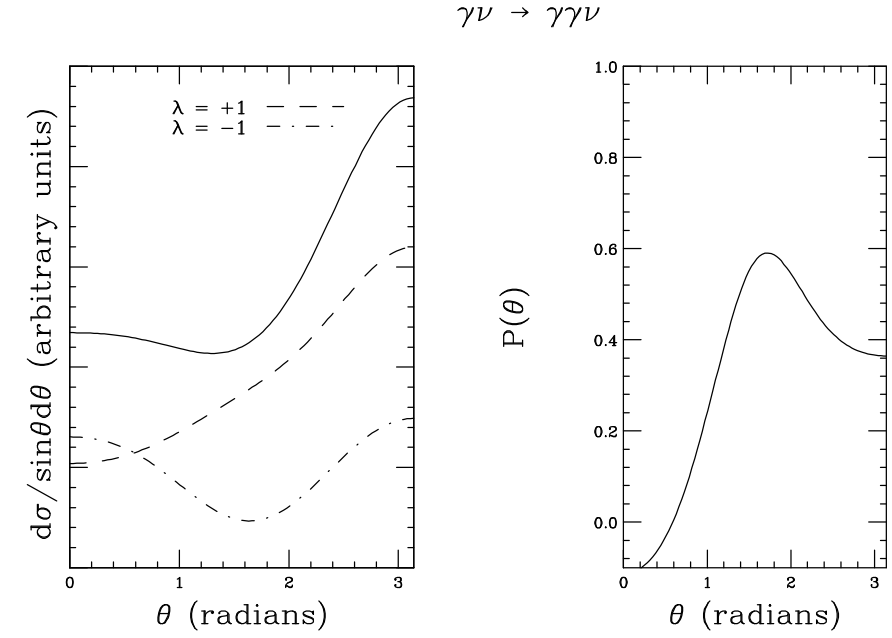

FIG. 2. The differential cross section $d \sigma / \sin \theta d \theta$ and polarization $P(\theta)$ are shown. In the left panel, the dashed line denotes the cross section for the production of a positive helicity photon, the dot-dashed line the cross section for the production of a negative helicity photon and the solid line is the sum of the two. 